\title{
An accelerated hybrid TLM-IE method for the investigation of shielding effectiveness
}

\author{
N. Fichtner and P. Russer \\ Institute for High Frequency Engineering, Technische Universität München, Arcisstr. 21, 80333 München, Germany
}

\begin{abstract}
A hybrid numerical technique combining timedomain integral equations (TD-IE) with the transmission line matrix (TLM) method is presented for the efficient modeling of transient wave phenomena. This hybrid method allows the full-wave modeling of circuits in the time-domain as well as the electromagnetic coupling of remote TLM subdomains using integral equations (IE). By using the integral equations the space between the TLM subdomains is not discretized and consequently doesn't contribute to the computational effort. The cost for the evaluation of the time-domain integral equations (TD-IE) is further reduced using a suitable planewave representation of the source terms. The hybrid TDIE/TLM method is applied in the computation of the shielding effectiveness (SE) of metallic enclosures.
\end{abstract}

\section{Introduction}

Shielding effectiveness (SE) is a measure for spurious radiation leaking out of metallic enclosures which contain electronic circuitry and moreover it denotes the prevention from external radiation to penetrate into an enclosure (Paul, 2006). The computation of SEs is usually a very complex task as the SE depends on various phenomena such as geometrical parameters, materials, apertures, contacts, cabling and connections. In order to account for all effects which may influence the SE an electromagnetic full-wave simulation is required which allows to incorporate all structural details and material properties. Further, it is desirable to compute the SE for a broad band of frequencies to identify forbidden or allowed operating frequency bands. Under this consideration the use of full-wave time-domain modeling techniques such as the finite-difference time-domain (FDTD) or the transmission line matrix (TLM) method is advantageous. Using either FDTD or TLM, complex 3-D structures of finite spatial extension can be modeled in the time-domain (TD). For SE measurements and simulations, however, the

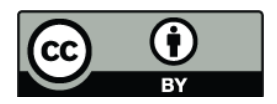

Correspondence to: N. Fichtner (fichtner@tum.de) electromagnetic fields some distance away from the enclosure are required (Paul, 2006). In this case integral equation (IE) based methods are preferable as the space between the source and the observation will not be discretized and hence does not contribute to the computational complexity. A hybrid technique therefore is proposed consisting of the TLM method in conjunction with TD integral equations (Fichtner and Russer, 2009). In the hybrid TD-IE/TLM method the circuitry together with the metallic enclosure and the aperture is modeled with TLM. The electric and magnetic fields leaving the metallic enclosure are probed at any time step and are used as source terms in the TD-IEs. From the TD-IEs, which are a TD version of the Stratton-Chu IEs (Hansen and Yaghjian, 1999; Tai, 2000), the fields at any position exterior of the TLM domain can be computed. In combination with the total-field/scattered-field (TF/SF) technique, spatially remote TLM subdomains can be coupled using TD-IEs (Fichtner and Russer, 2009). Moreover, a plane-wave time-domain (PWTD) algorithm based on the plane-wave representation of the radiated fields is employed to accelerate the TD-IE computation (Ergin et al., 1999; Chew et al., 2001).

\section{The hybrid TD-IE/TLM method}

\subsection{The total-field/scattered-field technique}

The hybrid TD-IE/TLM technique requires for the distinction between incident and scattered fields respectively inside a TLM domain. With the total-field/scattered-field (TF/SF) technique a partitioning between the total field, i.e. incident plus scattered field, and the scattered field only is possible. Consider a TLM domain $\Omega$ as shown in Fig. 1 in a crosssectional view. The inner region $\Omega_{1}$ contains all scatterers and sources and is surrounded by a second region $\Omega_{2}$ without any structure elements or sources. The boundary between the two regions is denoted as Huygens' surface $\partial \Omega_{12}$ (Fichtner and Russer, 2009; Taflove, 2000). Fields excited and/or scattered within region $\Omega_{1}$ will cross $\partial \Omega_{12}$ and are only outward propagating waves in region $\Omega_{2}$ as long as a absorbing boundary at the TLM domain boundary $\partial \Omega$ is used.

Published by Copernicus Publications on behalf of the URSI Landesausschuss in der Bundesrepublik Deutschland e.V. 


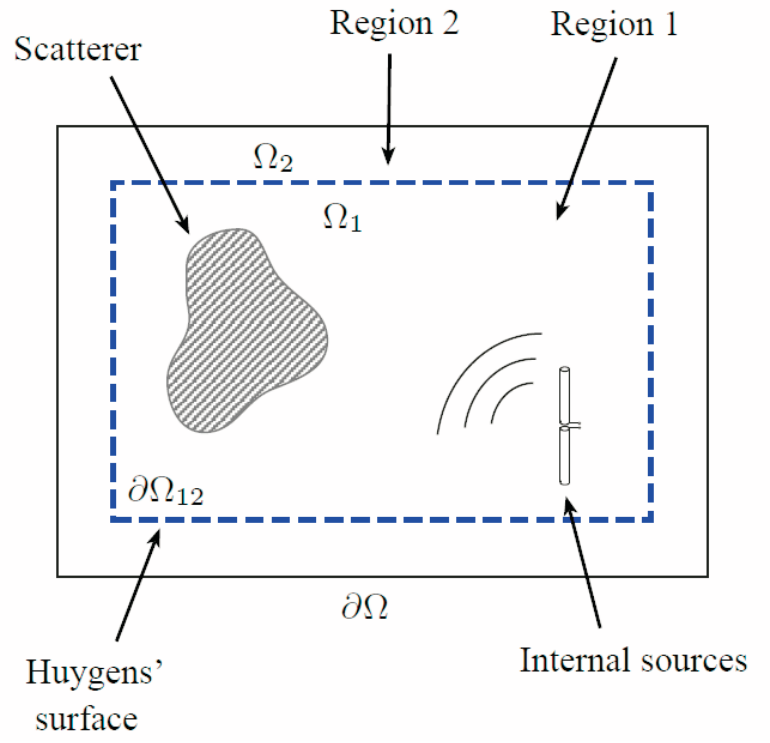

Fig. 1. TLM simulation domain $\Omega$ containing scatterers and source elements. $\Omega$ is subdivided in a total field region $\Omega_{1}$ and scattered field region $\Omega_{2}$. The dashed line indicates the Huygens' surface $\partial \Omega_{12}$.

In all simulations a perfectly matched layer (PML) boundary with 12 layers and a geometrical conductivity profile is used (Fichtner and Russer, 2009; Taflove, 2000). Consequently, the fields in region $\Omega_{2}$ can be used for the computation of the radiated near-fields and far-fields as well as for the coupling of different TLM domains (Fichtner and Russer, 2009).

Using the TF/SF technique it is also possible to excite arbitrary wave forms within region $\Omega_{1}$. These waves can either be analytically precomputed, e.g. for the excitation of arbitrary plane-waves (Taflove, 2000) or fields which originate from other remote TLM domains (Lindenmeier et al., 1999; Fichtner and Russer, 2009). For the one-side excitation of incident waves, a modified TLM connection operator has been derived (Fichtner and Russer, 2009). With ${ }_{k} \boldsymbol{E}_{l, m, n}^{\mathrm{inc}}$ and ${ }_{k} \boldsymbol{H}_{l, m, n}^{\mathrm{inc}}$ as the incident electric and magnetic fields, the modified TLM connection operator is written as

$$
\begin{aligned}
{ }_{k} \boldsymbol{a}_{l, m, n} & =\boldsymbol{\Gamma} \cdot{ }_{k} \boldsymbol{b}_{l, m, n} \\
& +\frac{1}{2}\left(\boldsymbol{\Gamma}_{E \cdot{ }_{k}} \boldsymbol{E}_{l, m, n}^{\mathrm{inc}}+\boldsymbol{\Gamma}_{H \cdot{ }_{k}} \boldsymbol{H}_{l, m, n}^{\mathrm{inc}}\right) .
\end{aligned}
$$

The connection matrices for the electric and the magnetic fields are

$$
\Gamma_{E}=\left[\begin{array}{lll}
\boldsymbol{A} & \mathbf{0} & \mathbf{0} \\
\mathbf{0} & \boldsymbol{A} & \mathbf{0} \\
\mathbf{0} & \mathbf{0} & \boldsymbol{A}
\end{array}\right], \quad \boldsymbol{A}=\Delta l\left[\begin{array}{cccc}
1 & -1 & 0 & 0 \\
-1 & 1 & 0 & 0 \\
0 & 0 & 1 & -1 \\
0 & 0 & -1 & 1
\end{array}\right]
$$

and

$$
\Gamma_{H}=\left[\begin{array}{ccc}
\boldsymbol{B} & \mathbf{0} & 0 \\
\mathbf{0} & \boldsymbol{B} & \mathbf{0} \\
\mathbf{0} & \mathbf{0} & \boldsymbol{B}
\end{array}\right], \quad \boldsymbol{B}=Z_{0} \Delta l\left[\begin{array}{cccc}
1 & -1 & 0 & 0 \\
1 & -1 & 0 & 0 \\
0 & 0 & -1 & 1 \\
0 & 0 & -1 & 1
\end{array}\right] .
$$

With Eqs. (1) to (3) it is possible to excite fields only within region 1 . No fields in region 2 will be excited as required for the hybrid TD-IE/TLM method. For vanishing incident fields Eq. (1) reduces to the standard TLM connection operator $\Gamma$ as required.

\subsection{Coupling integrals}

In numerical field computations the surface equivalence principle can be employed to compute the radiated electromagnetic fields everywhere in space from the knowledge of the field distribution on an arbitrary closed surface $\partial V$, which encloses all source elements (Balanis, 1997; Taflove, 2000). Consequently, the radiated near-fields or far-fields can be computed for simulation domains of finite extension (Taflove, 2000). The TD version of the Stratton-Chu IE using exterior differential forms is given by

$$
\begin{aligned}
\mathcal{E}(\boldsymbol{x}, t)= & \left.\mu \frac{\partial}{\partial t} \int_{\partial V^{\prime}} \mathcal{I} \wedge(\mathrm{n}\lrcorner \mathrm{n} \wedge \frac{\mathcal{H}\left(\boldsymbol{x}^{\prime}, t^{\prime}\right)}{4 \pi r}\right) \\
& -\int_{\partial V^{\prime}}\left(\star\left(\mathcal{R}^{\prime} \wedge \mathcal{I}\right)\right) \wedge \frac{\mathcal{E}\left(\boldsymbol{x}^{\prime}, t^{\prime}\right)}{4 \pi r^{3}} \\
& -\int_{\partial V^{\prime}} \frac{\partial}{\partial t^{\prime}}\left(\star\left(\mathcal{R}^{\prime} \wedge \mathcal{I}\right)\right) \wedge \frac{\mathcal{E}\left(\boldsymbol{x}^{\prime}, t^{\prime}\right)}{4 \pi r^{2} c} \\
& +\int_{\partial V^{\prime}} \mathcal{R} \wedge \frac{\star \mathcal{E}\left(\boldsymbol{x}^{\prime}, t^{\prime}\right)}{4 \pi r^{3}} \\
& +\int_{\partial V^{\prime}} \frac{\partial}{\partial t^{\prime}} \mathcal{R} \wedge \frac{\star \mathcal{E}\left(\boldsymbol{x}^{\prime}, t^{\prime}\right)}{4 \pi r^{2} c}
\end{aligned}
$$

where $\mathcal{I}$ is the unit double-form

$\mathcal{I}=\left(\mathrm{d} x \mathrm{~d} x^{\prime}+\mathrm{d} y \mathrm{~d} y^{\prime}+\mathrm{d} z \mathrm{~d} z^{\prime}\right)$.

(Details on exterior differential forms can be found in Russer (2006)). By $\mathcal{R}$ and $\mathcal{R}^{\prime}$ the distance 1 -form $\mathcal{R}=\left(x-x^{\prime}\right) \mathrm{d} x+$ $\left(y-y^{\prime}\right) \mathrm{d} y+\left(z-z^{\prime}\right) \mathrm{d} z$ is denoted whereas primed or unprimed differentials are used respectively. The unit 1-form $n$ in Eq. (4) is orthogonal to the considered plane of the Huygens' surface and is oriented outwards. An expression for the radiated magnetic field analog to Eq. (4) follows from the principle of duality.

A time and space discrete version of Eq. (4) and its dual is obtained by expanding the fields into two-dimensional pulsefunctions of edge length $\Delta l$ and applying point-matching. $\Delta l$ is identical to the TLM cell length in order to use directly the fields obtained from the TLM computation. After several 
step, which will not be outlined here, one obtains the compact representation

$$
\begin{aligned}
{ }_{k} \mathcal{E}(\boldsymbol{x})=\sum_{i=1}^{6} \sum_{j} & {\left[\boldsymbol{L}_{\mathcal{E} \mathcal{E} \cdot k^{\prime}} \mathcal{E}\left(\boldsymbol{x}_{j}\right)\right.} \\
& \left.+\boldsymbol{L}_{\mathcal{E} \mathcal{H} \cdot k^{\prime}} \mathcal{H}\left(\boldsymbol{x}_{j}\right)\right], \\
{ }_{k} \mathcal{H}(\boldsymbol{x})=\sum_{i=1}^{6} \sum_{j} & {\left[\boldsymbol{L}_{\mathcal{H} \mathcal{E} \cdot k^{\prime}} \mathcal{E}\left(\boldsymbol{x}_{j}\right)\right.} \\
& \left.+\boldsymbol{L}_{\mathcal{H} \mathcal{H} \cdot k^{\prime}} \mathcal{H}\left(\boldsymbol{x}_{j}\right)\right] .
\end{aligned}
$$

The time index $k^{\prime}$ is the retarded discrete time given by $k^{\prime}=k-\left|\boldsymbol{x}-\boldsymbol{x}_{j}\right| / c \Delta t$ where $\boldsymbol{x}_{j}$ is the source position and $\boldsymbol{x}$ is the observation position. The operators $\boldsymbol{L}$ are computed from Eq. (4) and consist of discrete temporal and spatial derivatives. From Eq. (6) the field at position $\boldsymbol{x}$ is computed from the retarded field values on a closed Huygens' surface $\partial V$ (see Fig. 1). In the hybrid TD-IE/TLM method the outgoing fields are probed on a closed Huygens' surface which is located within region $\Omega_{2}$. Using the probed fields as source terms, the radiated fields outside of the TLM domain $\Omega$ can be computed from Eq. (6). The radiated fields are used as excitation within a second TLM subdomain by using the TF/SF technique and the modified connection operator (1). Consequently, two or more TLM subdomains are electromagnetically coupled without discretizing the empty space between the subdomains.

\section{The plane-wave time-domain algorithm}

The dominant cost in the hybrid TD-IE/TLM method results from the computation of Eq. (6) which scales with $\mathcal{O}\left(N_{s}^{2} N_{t}\right)$. The number of basis-functions which constitute $\partial \Omega_{12}$ is given by $N_{s}$ and $N_{t}$ is the number of considered time-steps. A more efficient computation scheme is obtained if several source elements are first grouped together and in the following the contribution of every group is considered individually. This is basically done in the fast multipole method (FMM) used for the acceleration of frequency domain (FD) moment method (MoM) implementations (Chew et al., 2001). A TD analog of the FMM, which uses a planewave expansion for the representation of the outgoing fields, has been developed by Ergin et al. (1999); Chew et al. (2001). The plane-wave time-domain (PWTD) algorithm employs a Whittaker-type field representation of the form

$$
\begin{aligned}
\tilde{u}_{i}(\boldsymbol{x}, t) & =-\frac{1}{8 \pi c} \frac{\partial}{\partial t} \int \mathrm{d} \Omega \int_{V} \mathrm{~d} \boldsymbol{x}^{\prime} q_{i}\left(\boldsymbol{x}^{\prime}, t^{\prime}\right) \\
& =u_{i}(\boldsymbol{x}, t)-\int_{V} \mathrm{~d} \boldsymbol{x}^{\prime} \frac{q_{i}\left(\boldsymbol{x}^{\prime}, t+r / c\right)}{4 \pi r}
\end{aligned}
$$

where $\int \mathrm{d} \Omega$ is the area integral over the unit sphere. The unit direction vector is given by $\boldsymbol{k}=\boldsymbol{e}_{x} \sin \theta \cos \phi+\boldsymbol{e}_{y} \sin \theta \sin \phi+$

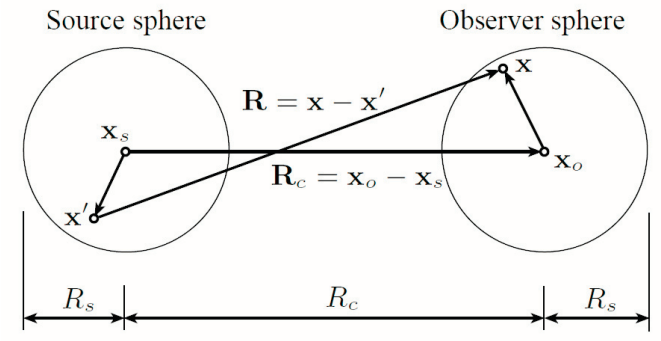

Fig. 2. Grouping of the source and observation positions inside two spheres of finite spatial extension.

$\boldsymbol{e}_{z} \cos \theta$ and $t^{\prime}$. The quantities $\tilde{u}_{i}(\boldsymbol{x}, t), u_{i}(\boldsymbol{x}, t)$ and $q_{i}(\boldsymbol{x}, t)$ are arbitrary field and source components respectively. In Eq. (7b) the second term on the right-hand side is anti-causal and can be eliminated by using proper time-gating techniques as outlined in Ergin et al. (1999); Chew et al. (2001).

Starting with Eq. (7) it is possible to derive a three-stage implementation for the summation in Eq. (6). According to Fig. 2 the distance vector $\boldsymbol{R}$ between the source and observation positions is first rewritten as

$\boldsymbol{R}=\boldsymbol{x}-\boldsymbol{x}^{\prime}=\left(\boldsymbol{x}-\boldsymbol{x}_{o}\right)+\boldsymbol{R}_{c}-\left(\boldsymbol{x}^{\prime}-\boldsymbol{x}_{s}\right)$

with $\boldsymbol{R}_{c}=\boldsymbol{x}_{o}-\boldsymbol{x}_{s}$. In the first stage of the PWTD algorithm the source distribution is mapped onto outgoing plane-waves propagating in all directions. This operation is denoted as Slant-stack transform (SST) and is given by

$q_{i}^{\text {out }}(\boldsymbol{k}, t)=\int_{V} d \boldsymbol{x}^{\prime} q_{i}\left(\boldsymbol{x}^{\prime}, t+\frac{\boldsymbol{k} \cdot\left(\boldsymbol{x}^{\prime}-\boldsymbol{x}_{s}\right)}{c}\right)$.

In Eq. (9) the time-dependent source distribution $q_{i}\left(\boldsymbol{x}^{\prime}, t\right)$ is represented by outgoing rays in the $\boldsymbol{k}$-th direction. In the second stage the rays leaving the source sphere are translated onto the observation sphere via the convolution operation

$q_{i}^{\text {in }}(\boldsymbol{k}, t)=\mathcal{T}\left(\boldsymbol{k}, \boldsymbol{x}_{c}, t\right) * q_{i}^{\text {out }}(\boldsymbol{k}, t)$

where $\mathcal{T}\left(\boldsymbol{k}, \boldsymbol{x}_{c}, t\right)$ is the diagonal translation operator

$\mathcal{T}\left(\boldsymbol{k}, \boldsymbol{x}_{c}, t\right)=-\frac{1}{8 \pi c} \frac{\partial}{\partial t} \delta\left[t-\frac{\boldsymbol{k} \cdot \boldsymbol{R}_{c}}{c}\right]$.

The incident rays depend on the direction of arrival $\boldsymbol{k}$ and time $t$. In the third and last step the incoming rays $q_{i}^{\text {in }}(\boldsymbol{k}, t)$ are projected onto the observation positions using

$\tilde{u}_{i}(\boldsymbol{x}, t) \int d \Omega q_{i}^{\text {in }}\left(\boldsymbol{k}, t-\frac{\boldsymbol{k} \cdot\left(\boldsymbol{x}-\boldsymbol{x}_{o}\right)}{c}\right)$.

In summary for a compact set of source and observation positions each with finite spatial extension the evaluation of Eq. (6) is accelerated by concatenation of all source elements, which are in case of the hybrid TD-IE/TLM method the fields ${ }_{k^{\prime}} \mathcal{E}\left(\boldsymbol{x}^{\prime}\right)$ and ${ }_{k^{\prime}} \mathcal{H}\left(\boldsymbol{x}^{\prime}\right)$ on the Huygens' surface. After the translation operation (10) a de-concatenation (12) is 


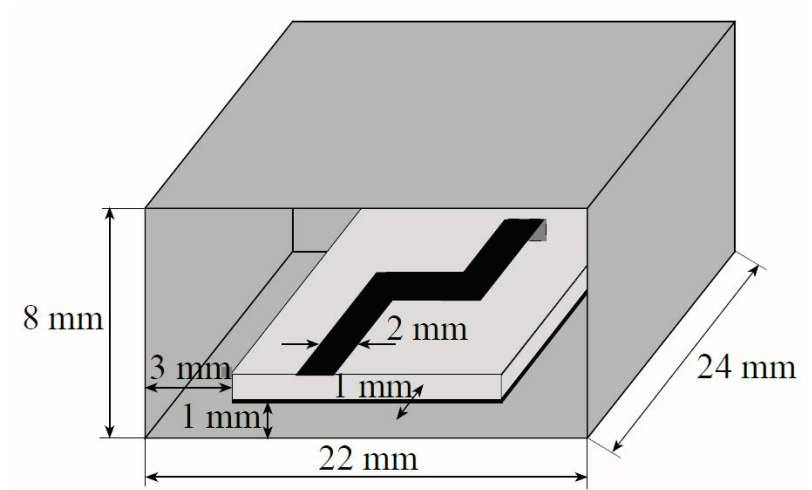

Fig. 3. Dimensions of the microstrip line inside the metallic enclosure.

applied. It can be shown that using the PWTD technique outlined between Eqs. (7) to (12) the computation of the TD-IEs scales with $\mathcal{O}\left(N_{s}^{1.5} N_{t} \log N_{s}\right)$. The acceleration is basically achieved by replacing the convolution in Eq. (10) with a multiplication whereas first the terms $\mathcal{T}\left(\boldsymbol{k}, \boldsymbol{x}^{\prime}, t\right)$ and $q_{i}^{\text {out }}(\boldsymbol{k}, t)$ are Fourier-transformed. After multiplication an inverse Fourier transform is applied. More details on the PWTD algorithm can be found in Ergin et al. (1999); Chew et al. (2001).

\section{Simulation results}

\subsection{Shielding effectiveness (1)}

The hybrid TD-IE/TLM method provides an efficient tool for the simulation and analysis of shielding problems. A measure for the shielding performance of enclosures and housings is the shielding effectiveness (SE), defined as

$\mathrm{SE}=20 \cdot \log _{10}\left|\frac{E_{0}}{E_{S}}\right|$,

where $E_{0}$ is the measured or simulated electric field without shield and $E_{S}$ is the electric field when the shielding is used (Paul, 2006).

In a first test case a microstrip line with two $90^{\circ}$ bends and a short at the end is placed inside a metallic enclosure. The microstrip line inside the metallic enclosure is shown in Fig. 3. The $90^{\circ}$ bends of the line are not compensated using mitered bends and hence spurious fields are excited at the bends. The microstrip line structure is excited using a waveguide port with a modulated Gaussian pulse defined between the frequencies 5 and $20 \mathrm{GHz}$. From the short the main part of the incident wave is reflected and leaking out of the circuit.

Additional to the metallic box as shown in Fig. 3 a front panel will be used to reduce field leakage out of the enclosure. Two different configurations for the front panel are investigated, which are shown in Fig. 4 (a) and (b). The front

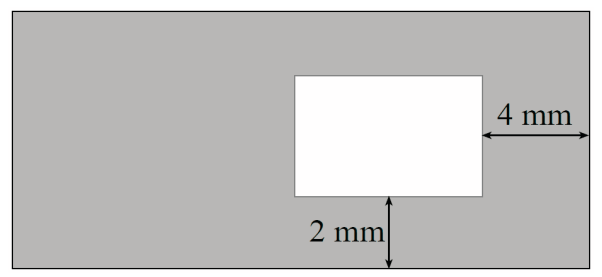

(a)

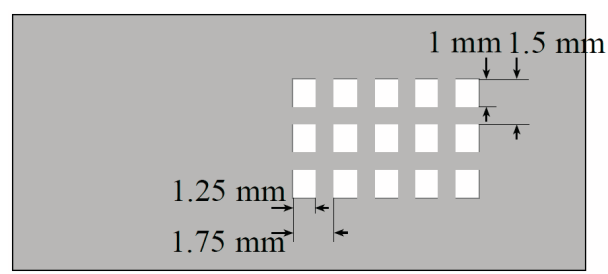

(b)

Fig. 4. Different configurations for the front panel of the enclosure. (a) exhibits a large air inlet of width $8 \mathrm{~mm}$ and height $4 \mathrm{~mm}$. (b) consits of several small air inlets.

panel in (a) exhibits a large air inlet of $8 \mathrm{~mm}$ width and $4 \mathrm{~mm}$ height and panel (b) has several smaller air inlets. Both air inlets (a) and (b) may serve as airflow apertures.

The electric field is measured $25 \mathrm{~cm}$ in front of the panels. In case of the TD-IE/TLM simulation the microstrip line and the enclosure is simulated with TLM whereas a Huygens' surface close to the box is used to probe the electric and magnetic fields leaking out from the enclosure. These fields are used as sources in the TD-IE computation (6). The Huygens' surface $\partial V$ is embedded within the source sphere as shown in Fig. 2. As the radiated fields at one position are required only, the first two steps of the PWTD algorithm are needed.

As a reference a CST simulation of the identical structure has been performed. The shielding effectiveness (SE) from 5 to $20 \mathrm{GHz}$ for the two panel configurations are shown in Fig. 5. For panel configuration (a) SE values between 0 and $60 \mathrm{~dB}$ are achievable. At some frequencies, in particular at $16.5 \mathrm{GHz}$ the SE is well below zero. In this case the air inlets even enhance field leakage as they operate as aperture antennas (Paul, 2006). Using panel (b) the SE is about $50 \mathrm{~dB}$ higher in comparison to panel (a). The SE characteristic with respect to frequency of panel (b) is almost identical to panel (a) except for a positive $50 \mathrm{~dB}$ offset.

The accordance between CST and TD-IE/TLM simulated results is very good for all considered frequencies. As in the TD-IE/TLM method the space between box and sense point is not discretized the hybrid method turns out to be approximately five times faster in comparison to a CST simulation when the same machine is used, where all the space between enclosure and measurement point is meshed. 


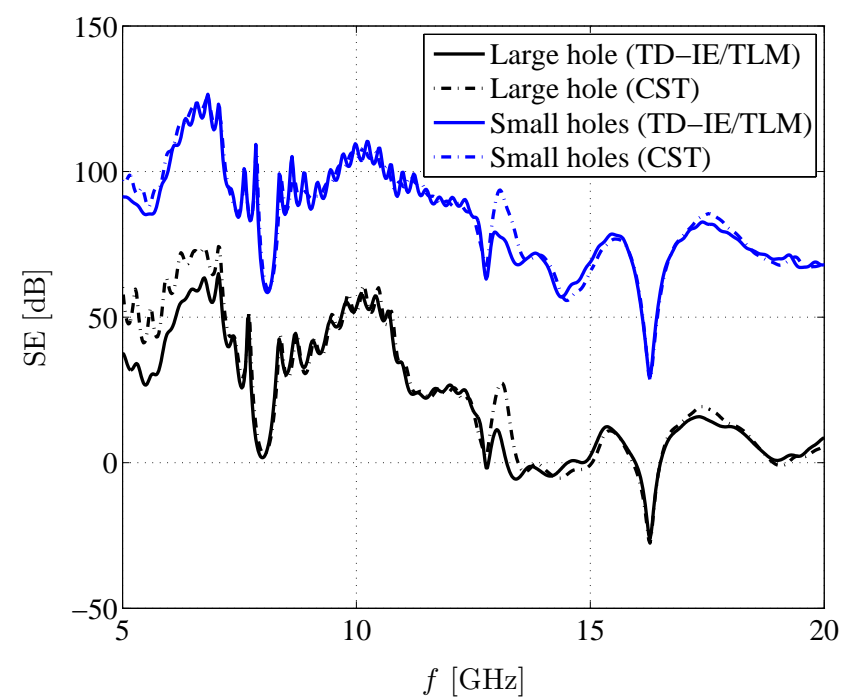

Fig. 5. TD-IE/TLM and CST computed shielding effectiveness (SE) for the two front panel configurations of Fig. 4.

\subsection{Shielding effectiveness (2)}

A further example concerning the shielding effectiveness is on the prevention of external radiation to penetrate into a metallic enclosure whereas different air inlet configurations are used. For this purpose a metallic box is considered which is irradiated by a broadband antenna. As antenna a planar bowtie will be used which can be modeled straightforward in TLM. The bowtie antenna geometry is shown in Fig. 6 . For the excitation of the antenna an electric field oriented in $x$-direction is impressed at its center. A modulated Gaussian pulse defined between the frequencies 1 and $5 \mathrm{GHz}$ will be used for the excitation. Usually such investigations are performed in the far-field (Paul, 2006). As the limit between near-field and far-field, the Fraunhofer distance $d=2 D^{2} / \lambda$ is considered, where $D$ is the maximum overall antenna dimension and $\lambda$ is the wavelength of interest (Balanis, 1997). For a frequency of $f=5 \mathrm{GHz}$ and a maximum antenna dimension of $D=95 \mathrm{~mm}$, given from the bowtie diagonal, the Fraunhofer distance $d$ is $300 \mathrm{~mm}$. In order to have some additional space between box and antenna the separation is set to $S=430 \mathrm{~mm}$.

The complete simulation scenario is shown in Fig. 7. The TLM subdomain $\Omega_{1}$ with the dimension $20 \times 20 \times 20$ cells contains the planar bowtie antenna of Fig. 6. The metallic box is modeled within a second TLM subdomain $\Omega_{1}^{\prime}$ of dimension $41 \times 41 \times 31$ cells. In both TLM subdomains the discretization $\Delta l=2.5 \mathrm{~mm}$ is used. The box has a square front with a side length of $105 \mathrm{~mm}$ and is $130 \mathrm{~mm}$ in depth. The two TLM subdomains in Fig. 7 show only the total-field regions. This means that the regions $\Omega_{1}$ and $\Omega_{1}^{\prime}$ are in addition surrounded by a scattered-field domain $\Omega_{2}$ and $\Omega_{2}^{\prime}$ respectively, whereas each domain is four TLM cells in width.

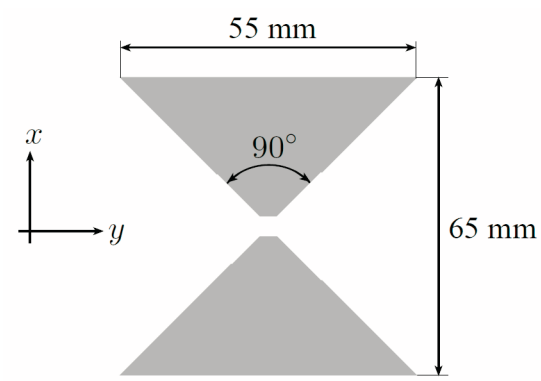

Fig. 6. Geometry of planar bowtie antenna.

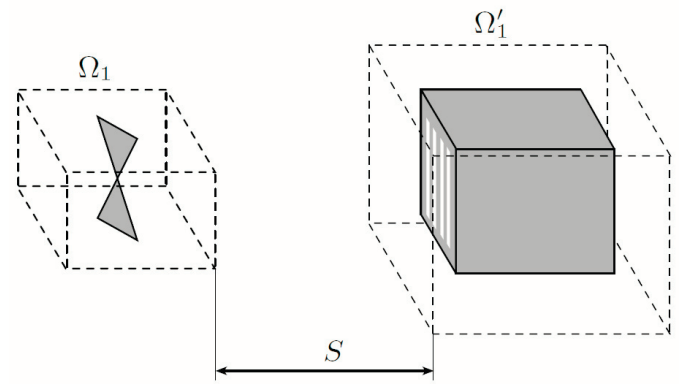

Fig. 7. TLM simulation scenario for the investigation of the shielding effectiveness of a metallic box irradiated by a planar bowtie antenna. The distance between the two TLM subdomain $\Omega_{1}$ and $\Omega_{1}^{\prime}$ is $S=430 \mathrm{~mm}$.

These scattered-field domains are not explicitly shown in Fig. 7. The TLM domains $\Omega_{1}$ and $\Omega_{1}^{\prime}$ are embedded within the source and observation sphere respectively of the PWTD algorithm.

For the front plane of the metallic box four different configurations of air inlets are investigated. The front planes are shown in Fig. 8. For configuration (a) the inlets are square and have a width of $15 \mathrm{~mm}$. The separation between the inlets is $10 \mathrm{~mm}$. In configuration (b) the width of the square inlets and the separation is identical to $5 \mathrm{~mm}$. In case of configurations (c) and (d) the horizontal and vertical stripes have a width of $5 \mathrm{~mm}$ and a length of $65 \mathrm{~mm}$.

In this analysis the excitation of resonant modes inside the metallic enclosure caused be the fields penetrating into the enclosure, i.e. the type of modes and their amplitudes are of interest. The electric field radiated from the bowtie antenna is polarized in $x$-direction. Thus, the $E^{x}$ component inside the box is measured 10 cells behind the front panel. The magnitude spectra of the probed electric field using the four front plane configurations (a) to (d) are shown in Fig. 9. In case of configuration (a) the signal amplitudes are very high because of the strong coupling. The interior of the box is only weakly shielded from external radiation if large air inlets are used. Moreover, the resonating modes exhibit high quality factors as can be seen from the sharp peaks at the resonant frequencies. This indicates that the resonating modes 


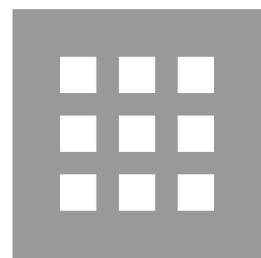

(a)

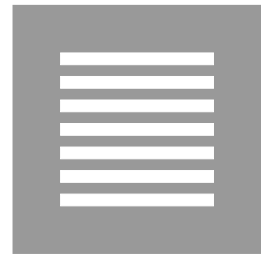

(c)

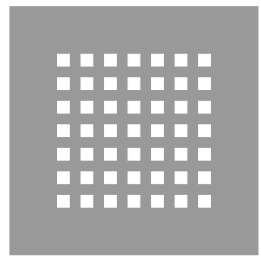

(b)

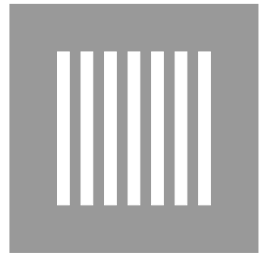

(d)
Fig. 8. Air inlet configurations for the shielding effectiveness investigations on the irradiated metallic enclosure.

are only weakly disturbed by the inlets. From the magnitude spectrum in Fig. 9 it can be deduced that the highest shielding effect can be achieved if either the small inlets of configuration (b) or the $x$-directed slots of configuration (d) are used. In case of (b) the shielding is so strong that almost no resonances are excited within the box. The slots of configuration (d) behave like a polarization filter and prevent most of the radiation from penetration into the box. The situation is different in case of configuration (c). Here the slots are oriented orthogonal to the polarization of the incoming electric field and thus the fields can easily penetrate into the box. Though the formation of resonating modes is rather weak. It can be shown that the lowest order resonating modes are of $\mathrm{TE}_{10 l}$-type with an electric field in $x$-direction. Due to the orientation of the metallic bars in case of configuration (c) the electric fields of the $\mathrm{TE}_{10 l}$ modes can easily leak out from the metallic box which explains the low quality factors.

\section{Conclusion}

A hybrid TD-IE/TLM method using the plane-wave timedomain technique has been presented for the fast and efficient modeling of typical shielding problems. Circuits together with enclosures are modeling with TLM and the radiated fields in the near-as well as in the far-field are obtained from TD integral equations. The computational burden for the evaluation of the integral equations is lowered by using a suitable plane-wave representation. In case of the presented SE simulations the computational effort is lowered by a factor of 3 to 5 if the hybrid TD-IE/TLM method is used compared with a pure TLM simulation. Using the PWTD algorithm further $20 \%$ reduction in computational cost are achievable.

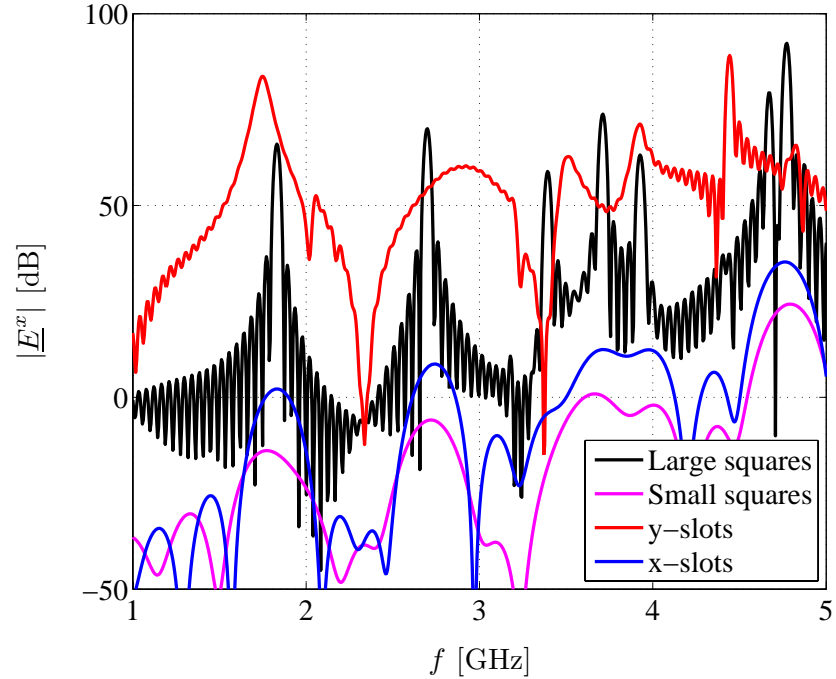

Fig. 9. Magnitude spectrum of the $E^{x}$ field components measured inside a metallic box for the different front panel configurations of Fig. 8 .

Acknowledgements. This work was supported by the Deutsche Forschungsgemeinschaft (DFG).

\section{References}

Balanis, C.: Antenna Theory - Analysis and Design, John Wiley \& Sons, 2nd edn., 1997.

Chew, W., Michielssen, E., Song, J. M., and Jin, J. M.: Fast and Efficient Algorithms in Computational Electromagnetics, Artech House, Norwood, MA, USA, 2001.

Ergin, A., Shanker, B., and Michielssen, E.: The Plane-Wave Time Domain Algorithm for the Fast Analysis of Transient Wave Phenomena, IEEE Trans. Antennas Propagat. Magazine, 41, 39-52, 1999.

Fichtner, N. and Russer, P.: A Total-Field/Scattered-Field Technique Applied for the TLM-Integral Equation Method, 2009 IEEE MTT-S Int. Microwave Symp. Dig, 325-328, 2009.

Hansen, T. and Yaghjian, A.: Plane-Wave Theory of Time-Domain Fields, IEEE Press, 1999.

Lindenmeier, S., Pierantoni, L., and Russer, R.: Hybrid Space Discretization - Integral Equation Methods for Numerical Modeling of Transient Interference, 1999, IEEE Trans. Electromagn. Compatibility, 41, 425-430, 1999.

Paul, C.: Introduction to Electromagnetic Compatibility, Wiley Series in Microwave and Optical Engineering, John Wiley \& Sons, New York, 2nd edn., 2006.

Russer, P.: Electromagnetics, Microwave Circuit and Antenna Design for Communication Engineering, Artech House, 2nd edn., 2006.

Taflove, A.: Computational Electrodynamics - The Finite Difference Time-Domain Method, Artech House, Boston, 2nd edn., 2000.

Tai, C.-T.: Direct Integration of Field Equations, Progress in Electromagn. Research, 28, 339-359, 2000. 